\title{
Situation-aware Building Information Models for Next Generation Building Management Systems
}

\author{
Ovidiu Noran ${ }^{1,2 \mathbb{D}^{\mathrm{a}}}$, Peter Bernus ${ }^{1} \mathbb{( \mathbb { D }}^{\mathrm{b}}$ and Sorin Caluianu ${ }^{2} \mathbb{E}^{\mathrm{c}}$ \\ ${ }^{1}$ IIIS Centre for Enterprise Architecture Research and Management - Griffith University, Brisbane, Australia \\ ${ }^{2}$ Faculty of Installations Engineering, Technical University of Constructions Bucharest, Romania
}

Keywords: Building Information Model, Building Management System of Systems, Decision Support Systems, Situational Awareness, Big Data, Data Warehousing, Decision Model, Situated Reasoning, Channel Theory.

\begin{abstract}
Technical advances in Information and Communication Technology have enabled the collection and storage of large amounts of data, rising hopes of improving asset decision-making and related building management support systems. It appears however that the gap between the required decision-making knowledge and the actual useful information provided by current technologies appears to increase, rather than contract. Thus, often the multitude of patterns afforded by current data analytics techniques does not deliver a set of scenarios prone to effective decision making. This paper advocates a decision analytics solution featuring the use of Situated Logic to create 'narratives' providing adequate meaning to data analytics results, and the use of Channel Theory so as to support adequate situational awareness. This approach is also analysed in the context of a Building Management System-of-Systems paradigm, highly relevant to the emerging complex Clusters of Intelligent Buildings within Smart Cities, featuring collaborative decision-making centres and their associated decision support systems.
\end{abstract}
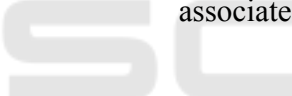

\section{INTRODUCTION}

Building information models (BIMs) are files typically containing proprietary formats and data which can be extracted, exchanged or networked to support decision-making within a Building Management System (BMS) for a built asset (Van Nederveen \& Tolman, 1992).

BIM can be used to enhance building decision making (Nowak, Ksiazek, Draps, \& Zawistowski, 2016), to coordinate projects (Rokoeei, 2015) and deliver sustainable building value (Fadeyi, 2017).

BIMs have an important role in reducing the fragmentation among professionals at each stage and across building delivery stages by providing a virtual repository that allows easy access to- and sharing of information and knowledge in real time. Thus, BIM also enhances interoperability, providing a platform for professionals to work in an integrated environment at any stage of the building delivery process and further on in the management phase within BMS (Santos, 2009).

Although traditionally focused more on the short term and real-time operation, BIMs and BMS are increasingly required to assist in medium- and long term asset planning, especially in the case of Intelligent Building Clusters in Smart Cities (Zhang et al., 2018). The scope of the models is also expected to expand from mainly energy consumption to all aspects of asset management so as to provide a holistic and integrated view (Teliceanu, Golovanov, Lazaroiu, \& Dumbrava, 2017; Wei \& Zhu, 2016). It must also be noted that nowadays, due to the increased complexity brought by numbers and variety of controlled parameters, BMS are increasingly taking on hybrid agent features, featuring human and machine components, beyond mere Human Machine Interface (HMI) within the BMS 'head end' (Forte Asset Services, 2019). All of the above endeavours essentially depend on the quality and relevant content of the information obtained from the collected data.

\footnotetext{
a (iD https://orcid.org/0000-0002-2135-8533

b(i) https://orcid.org/0000-0001-5371-8743

c(iD https://orcid.org/0000-0001-5961-4187
} 
Thus, Big Data (Li, Zhang, Hu, \& 17., 2017), the Liquid Enterprise (Bayler, 2016), Sensing Information Systems (Zdravković, Noran, \& Trajanović, 2014) and similar concepts hold the promise to provide all necessary decision-making information for building management in the adequate detail, quality and 'freshness' required. Conceptually, this endeavour comprises achieving the necessary capabilities to use the data to derive decision-making information in an efficient and effective manner, based on inferring knowledge that was not available (and attainable) before; nowadays, this assumes the presence and support of suitable data analytics.

The degree of success in supporting decisionmaking heavily depends on proper data synthesis and interpretation (Dibb, Meadows, \& Wilson, 2015). Thus, proper data analytics will effectively support decision analytics.

It is also becoming clear that finding new ways to correctly interpret complex data in context is necessary (SAS, 2015). For example, evidence-based medicine that relies on large scale data gathering through clinical trials and careful statistical analysis is now showing difficulties when the evidence gathered is applied in complex individual cases (Greenhalgh, Howick, \& Maskrey, 2014; Pope, 2003).

From the above, an obvious perspective is that when intending to use large amounts of gathered data to create useful decision-making information, one must carefully consider the information needs of the intended audience (e.g. management) and importantly, how the interpretation of data is influenced by context.

This paper intends to analyse the barriers in using data warehousing and big data approaches for BIM in order to support proper decision analytics manifested in effective decision-making within BMS.

\section{DATA TO INFORMATION: CONCEPTS AND APPROACHES}

\subsection{Building Management Systems and Their Information Needs}

Initially limited to operational and real time, Building Management Systems nowadays increasingly need to also make decisions on strategic and tactical levels. This endeavour can be reasoned about in relation to the information flow, usage and needs, and optimised using various types of models. In the following, the authors will use an example of mainstream systematic model of decision-making, namely the GRAI Grid (Doumeingts, Vallespir, \& Chen, 2003) (see Fig. 1). Fundamentally, this generic model identifies management, command and control tasks at various levels (identified via time spans called 'horizons') and the information flow between them.

The exogenous and endogenous information flows feeding the Manage, Command and Control centre in Fig. 1 illustrate the point that in order to make successful decisions it is necessary to satisfy the information needs of the management functions.

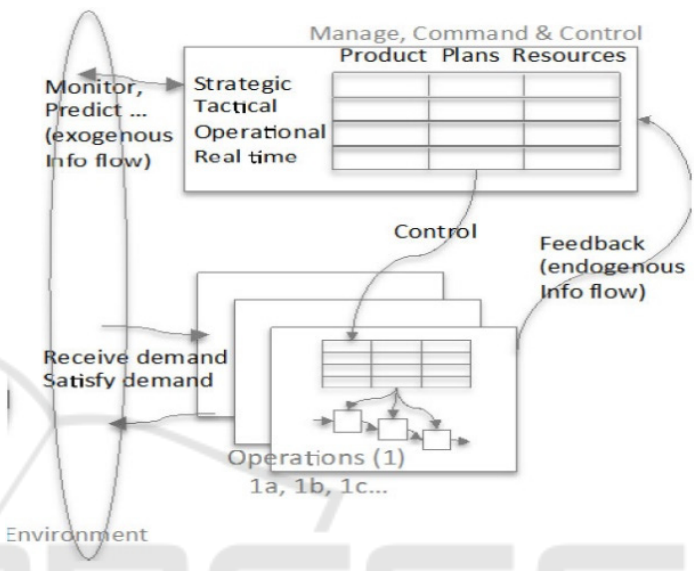

Figure 1: Information flows in a Basic mainstream decision making model: GRAI Grid (Doumeingts, Vallespir, \& Chen, 2003).

This means that the data gathered and analysed must be meaningful, properly aggregated (level of detail) and suitably expressed in order to meet the demands and competencies of each audience populating the decision centres at various horizons.

This is not a trivial task. To justify this point the authors refer to two main approaches to data analytics, namely data warehousing and big data.

\subsection{Data Warehousing and Big Data}

The concept of Data warehousing is relying on high quality clean and integrated data to enable the use of operational databases and other data repositories snapshots and build an interface enabling the analysis ('mining') in order to identify management-relevant information. Some authors (Inmon, Zachman, \& Geiger, 1997; Kimball, 1996) argue that such a facility could be built fast and in an affordable manner using existing databases and possibly transaction logs so as to gain management insight (Matte \& Rizzi, 2009; Satyanarayana, Srinivasu, Poorna Rao, \& Rikkula, 2010). The aim is to create a narrative that 
is characterising the present or predicted future situation and is essential for decision-making. After some initial success in creating meaningful insight for management, data warehousing displayed some notable failures to deliver on its promises (Adelman \& Moss, 2000).

'Big data' is a technology that uses traditional data analysis and machine learning techniques to derive useful interpretations based on large and varied data sources (Gandomi \& Haider, 2015; Marr, 2015). Brought forward by the technological advances in data gathering (cheaper and more intelligent Internetof-Things (IoT)-enabled sensors, cheaper and cloudbased storage etc.) and initially boasted as the solution to the problems where traditionally implemented data warehousing fell short, big data is still maturing and yet to make significant inroads in decision support (Horitaa, de Albuquerquea, Marchezinid, \& Mendiondoc, 2016; Kościelniaka \& Putoa, 2015). This is also due to its inherent dependence on machine learning algorithms that attempt to predict but cannot adequately explain the predictions, which is an essential factor in gaining human trust in decision support systems (Wang \& Benbasat, 2008).

\subsection{Shortcomings of Data Warehousing and Big Data}

A first drawback of the two concepts refers to the associated methodologies, which do not lay enough emphasis on understanding the fundamental information needs of the decision maker rather than rushing to data collection and interpretation (Seen \& Sinha, 2005).

In addition, there is minimal or inexistent correlation between internal- and external data sources (i.e., connecting the endogenous and the exogenous information flows - see Figure 1).

Further on, insufficient effort is put into realising what data is needed for being able to draw useful inferences, but is unavailable. Even the recent approaches proposing to limit the amount of sensor data used in situation assessment (e.g. by switching on- or off additional pre-stored sensor data sources) is in fact relying on the command and control to pinpoint what data should be taken into account to possibly change the narrative.

If the above deficiency is identified, then the need for data that is not available, but is deemed necessary, may become the source of additional data collection tasks; however, this can inadvertently result in poor data quality ((Hazen, Boone, Ezell, \& Jones-Farme, 2014; SAS, 2015)).
Another issue is the limited progress in transforming existing building management processes to produce the necessary data as a byproduct of the production (or service delivery) process, instead of requiring additional data entry (Hazen et al., 2014) (a main source of data quality issues as shown above).

There has been a tendency to disregard the collected data context (Corrigan, 2007), thus creating the danger of situation mis-identification without even being aware of having committed this mistake (Santanilla, Zhang, Althouse, \& Ayers, 2014).

Another important aspect is the typical reliance of big data technology on machine learning techniques producing models whose uncertainty cannot be adequately assessed and whose predictions cannot be adequately explained (Kendall \& Gal, 2017).

To conclude, the main shortcomings found are:

- On each decision-making level, one must correlate internal and external data.

- With the opportunity to collect and access very large amounts of building-related data, the typically low density of useful content (Li et al., 2017) makes it difficult to identify patterns that are useful for decision making (too many patterns identifiable by algorithms) - unless one uses heuristics (i.e., the result of prior learning) to discern what is relevant (note that the measure of relevance may change in time and with the current interpretation of data).

\section{BUILDING INFORMATION MODELS FOR EFFECTIVE DECISIONS}

\subsection{The OODA Loop as an Activity Network}

The tasks that appear in each type and level of decision-making and the feedback that can be used to inform the filters used to selectively observe reality might be studied using a model that explains how successful decisions are made. This model is part of the Observe, Orient, Decide and Act (OODA) Loop devised by John Boyd (Osinga, 2006).

Although some authors such as Benson \& Rotkoff (2011) understand OODA to be a strict sequence of tasks, this is in fact not true due to the feedback links inside the high level 'loop-like' structure that are responsible for learning and for decisions about the kind of filters necessary. Thus, OODA is in fact an activity network featuring rich information flows among its activities and the environment. 
A brief review of Boyd's OODA concept can be used to highlight potential development directions for data warehousing and/or big data methodology for decision support. Thus, decisions can be made by the management / command \& control system of a BMS, in any domain of action and on any level or horizon of management (i.e., strategic, tactical, operational and real-time, performing four interdependent tasks. The tasks that appear in each type and level of decision-making and the feedback that can be used to inform the filters used to selectively observe reality may be studied using a model that explains how successful decisions are made.

Note that this 'loop' is often misunderstood to be a strict sequence of tasks (Benson \& Rotkoff, 2011). OODA is not a strict loop, due to the feedback links inside the high level 'loop-like' structure that are responsible for learning and for decisions about the kind of filters necessary. Thus, in fact it is actually an activity network featuring rich information flows among the OODA activities and the environment.

This learning has the potential to result in decisions that also emphasize relevant gaps and thus initiate capability improvement efforts. Self-reflective management typically engages in such learning, comparing the behaviour of the external world and its requirements on the system (the future predicted action space) with the action space of the current system (including the current system's ability to sense, orient, decide and act). Note that here, the term 'action space' describes the set of possible outcomes reachable using the system's current resources (technical, human, information and financial).

The learning loop is in itself an OODA loop analogous to the one discussed above, although the ingredients are different and closely associated with strategic management (see Figure 2). Thus the OODA-style questions are in this case: a) what to observe, b) how to orient to become situation-aware and c) what is guiding the decision about what to do (within constraints, decision variables and possible actions) so as to be able to act. The action space of this strategic loop consists of transformational actions (company re-missioning, change of identity, business model change, capability development, complete metamorphosis, etc.).

Strategic self-reflection compares the current capabilities of the system to desired future capabilities, enabling management to decide whether the change will affect the system's capabilities (including decision making capabilities), the system's identity (re-missioning), or both. Note that the management may also decide to instead decommission that part of the system due to its inability to fully perform the system's mission.

Such transformations are typically implemented using a separate programme or project using a similar suitable iterative paradigm, such as the so-called Plan-Do-Check-Act (PDCA) loop (Lawson, 2006), possibly in a recursive manner (Schmidt, Elezi, Tommelein, \& Lindemann, 2014) e.g. for complexity control.

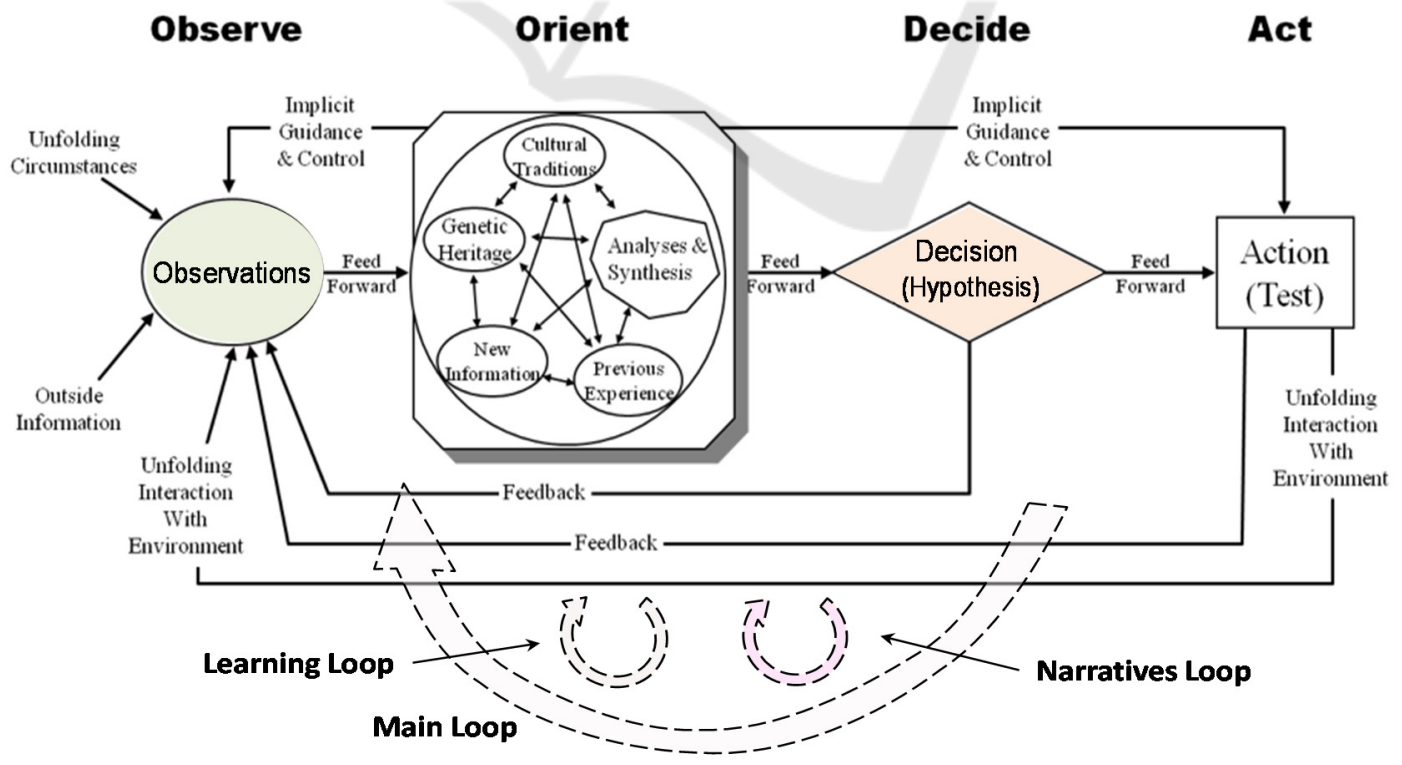

Figure 2: Extended OODA Loop as an activity network (based on (Fadok, Boyd, \& Warden, 1995)) featuring additional Learning and Narratives Loops. 


\subsection{Consequences for BMS Decision Support based on Data Warehousing and Big Data - Enabled BIM}

The above analysis implies that 'big data' (meaning the collective technologies and methods of data analysis and predictive analytics) has the potential to enable situational awareness (a condition of successful action) by delivering a plethora of previously unavailable domain-level facts and patterns relevant for decision-making. However, this data needs to be interpreted, which calls for a theory of situations resulting in a narrative of what is being identified or predicted. Without such a narrative, there is no true situational awareness or trust in the system, which can substantially limit the chances of effective action.

It is therefore argued that having the ability to gather, store and analyse large amounts of data using only algorithms is not a guarantee that the patterns thus found in data can be turned into useful and trustworthy information that forms the basis of effective decision-making, followed by appropriate action leading to measurable success.

Importantly, the process is similar the other way around: when interpreting available data, there can be multiple fitting narratives; unfortunately, it is quite difficult to choose the 'correct' one. In this case, adequate means of reasoning with incomplete information could help articulate a need for new data (or new types of data) that can resolve the ambiguity.

As a result of the above reasoning, the authors argue that supporting decision-making based on data warehousing using 'big data' requires the collection of a second level of data. This 'second level' is not meant to refer to particular facts, but rather to underpin the creation of an inventory of situation types, containing facts that must be true, facts that must be not true, as well as constraints and rules of corresponding causes and effects. These situation types can be considered models (or model prototypes) of the domain, which can be matched against findings on the observed data level.

Note that due to the changing nature of the Universe of Discourse, the above-mentioned situation types are also expected to evolve; therefore, one should not aim to design and / or construct a facility that relies on a completely predefined ontology of situation types. Rather, there is a need for a capability to continuously improve and extend this type of knowledge, including the development and learning of new types, which are not a specialisation of some known type. This is required in order to ensure that the 'world of situations' remains open, as described by Goranson and Cardier (2013).

In order to achieve adequate situation awareness for effective decision making, collected data needs to be filtered based on relevance ( $\mathrm{Li}$ et al., 2017; Szafranski, 1995), dictated by the possible situations of interest. However, as the current situation is typically not unambiguously known and changes as data gathered is interpreted, one will have to maintain a dynamic narrative (or set thereof) of the situation, which will continually adjust the data needs (Madden, 2012) as well as what needs to be filtered out, or be kept. This constitutes yet another OODA loop, applied to the set of narratives involved in the interpretation of data for decision making (see Narratives Loop in Figure 2).

\section{DESIGN PRINCIPLES FOR 'NEXT GENERATION' BMS DECISION MAKING}

On both existing and emergent system levels, decision making needs a timely and accurate narrative that looks behind the 'observables'. An essential aspect of agile and effective decision making (whether on strategic or tactical level) relies on the ability of the system in question to create and to continually update its situated insight, thus being able to deal with uncertainty.

\subsection{First Functional Principle: Employ Situated Reasoning}

Consider the following domain-level observations within the domain of building energy demand at cluster level and its coupling with complex integrated energy systems; O1: some buildings within an 'intelligent' cluster report increased levels of energy consumption; O2: occupants of some of the buildings within the cluster (some overlap with $\mathrm{O} 1$ ) report reduced thermal comfort, O3: there are no reports of malfunction. Then, O4: energy consumption goes to normal however O5: occupants still complain. Then later on, the situation $(\mathrm{O} 1-\mathrm{O} 5)$ repeats itself.

A domain-level theory could describe energy supply-demand rules in complex smart building clusters including e.g. energy consumption fluctuations depending on various factors.

While it is possible to build a model of the situation, it is quite difficult and computationally intensive to know all the intricacies (in space and time) of such complex interconnected systems within 
the building cluster (Frayssinet et al., 2018). More importantly, the information usable to address the situation with the right decision is incomplete. Therefore, one does not really know what really causes the increased energy consumption and thermal discomfort in the absence of well-identified malfunctions.

Typically, the Service Entity (SE) attempting to address the situation must be able to interpret past observed events and build types of situations (the candidate interpretations of the events described), each with their own logic and constraints. Thus, a competent SE would be familiar with a repertoire of situation types and their internal logic. The question is: which one of these can be used as the correct narrative of the observed events in this case?

The SE can interpret the current situation $\boldsymbol{S}$ based on known matching situation types $\boldsymbol{S}_{\mathrm{A}}-$ e.g. sensor measurement drift (e.g. due to low quality control or bugs in embedded software) in some batches and $\boldsymbol{S}_{\mathrm{B}}$, e.g. semantic communication problem between BMS-es within the cluster. Note that there may be a myriad of situation types $\boldsymbol{S}_{\mathrm{N}}$, some being variations of a general type.

The SE's Decision Suppot Sstem (DSS) can employ situated reasoning in order to find out what (perhaps very simple) additional fact/s would have to be discovered to disambiguate between $\boldsymbol{S}_{\mathrm{A}}$ and $\boldsymbol{S}_{\mathrm{B}}$ and thus be able to act appropriately.

While such avenues of action may seem rather simple for humans, the real issue is the potential automation and optimisation of such behaviour in BMS, based on interpretation of BIM data. In order to achieve this, one needs to employ a second functional princciple.

\subsection{Second Functional Principle: Channel Theory for Situation Awareness}

If situations are organised in types and their internal logic is known (like in the scenario in Section 4.1), then there exist possible 'channels' through which information in one situation type can be transferred (possibly in a lossy manner) to another situation type.

A recently popularised mathematical approach of the above is the category theoretic treatment of situation theory (Barwise \& Perry, 1998; Devlin, 2003; Goranson \& Cardier, 2013). The mechanism that allows the two levels (situation theory and domain level theory(ies)) to coexist is channel logic (Barwise \& Seligman, 2008) - according to which, given the category of situations representing situation types, there is a mapping that regulates the way complete lines of reasoning can be 'transplanted' from one situation type to another.

This transplanting works as follows: when there exists a logic in a known situation type $\boldsymbol{S}_{\mathrm{A}}$ and the facts suggest that the situation is of a related type $S_{\mathrm{B}}$, many (however typically not all) facts and inferences should also be valid in type $\boldsymbol{S}_{\mathrm{B}}$ (see Figure 3).

As a result, if we have a known situation of type $\boldsymbol{S}_{\mathrm{A}}$ with facts supporting this claim, and we only have scarce data about another situation type of interest (of type $\boldsymbol{S}_{\mathrm{B}}$ ), channel logic allows us to deduce the need for data that can be used to 'fill in the details' about this second situation of type $\boldsymbol{S}_{\mathrm{B}}$. The resulting mapping is a so-called morphism between categories and can be implemented using functional programming techniques. In Figure 3, this (info-) morphism is represented by the double-headed arrows shown carrying information (while possibly losing some, as previously stated) from one situation type to another type to another.

In the case described in section 4.1, this morphism may mean analysing particular sensor manufacturing data and/or simulating events on the BMS in question while observing the effects (as the original events appear to be of a transient and random nature, either due to random sensor drift or complex unwanted interaction within BMS software or even sensor embedded software).

The practical consequence is that the decision maker can use this analogical reasoning to come to valid conclusions in an otherwise inaccessible domain; should this not be possible, it allows to at least narrow down the need for specific data that can support a valid conclusion.

The above also illustrates in a simplified way the ability of the situation theoretic logic to infer that for decision making, there is a need for specific, but yet unavailable data that can disambiguate the interpretation of what is known at the time.

\section{CONCLUSIONS AND FURTHER WORK}

In the context of the increasing rate of change and the resulting flood of data, decisions, even in traditionally local and non-time-critical domains such as BMS will have increasingly far-reaching consequences and need to be promptly made - often, in real time.

The work presented in this paper can be used towards creating an ongoing situational awareness capability. The effective use of 'proven repertoires' nowadays increasingly depends on their fast, near-automated 


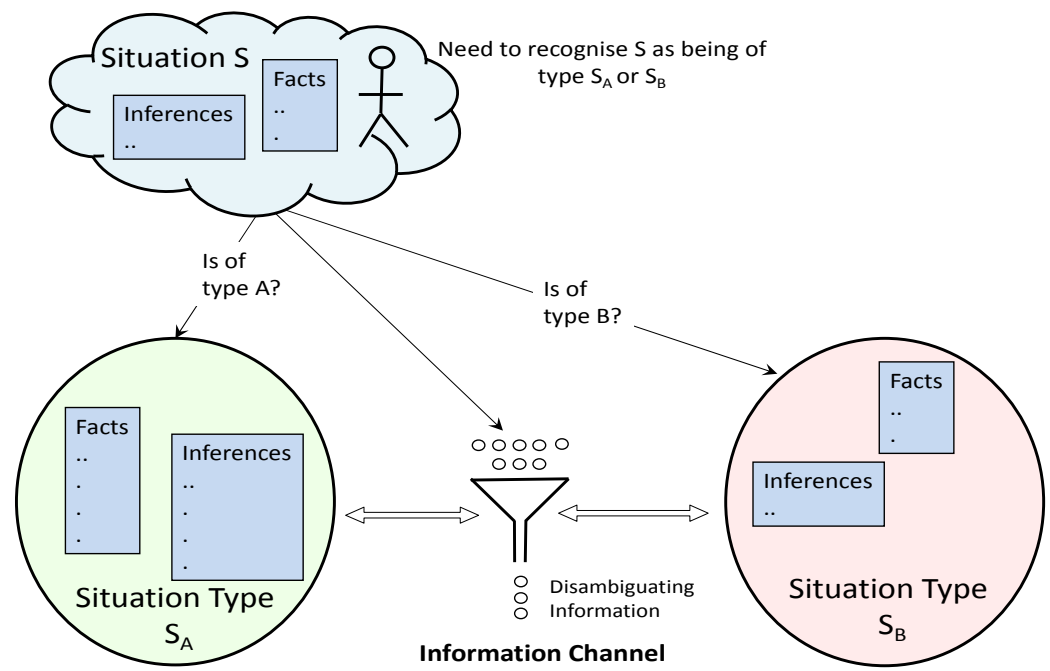

Figure 3: Simplification of Category Theoretic approach of Situation Theory (Noran \& Bernus, 2018).

deployment, which is traditionally based on human tacit skills and knowledge.

The paper has shown how novel paradigms can assist traditional data analytics approaches in order to achieve adequate BIM situation awareness and thus properly support decision-making within complex Building Management Systems-of-Systems.

Further work will continue to focus on the principles underpinning situation theory-based decision-making and related supporting technology. The results will be used to demonstrate their use in complex Building Management Systems-of-Systems within clusters of Intelligent Buildings present in the emerging Smart City paradigm.

\section{REFERENCES}

Adelman, S., \& Moss, L. (2000). Data Warehouse Risks in Data Warehouse Project Management: Addison WL

Barwise, J., \& Perry, J. (1998). Situations and Attitudes: Center for the Study of Language and Inf.

Barwise, J., \& Seligman, J. (2008). Information Flow: The Logic of Distributed Systems. In Cambridge Tracts in Theoretical Computer Science. Cambridge: Cambridge University Press.

Bayler, M. (2016). The Liquid Enterprise: How the network is transforming value, what it means for business, and what leadership needs to do about it. UK: Infinite Ideas.

Benson, K., \& Rotkoff, S. (2011). Goodbye, OODA loop: A complex world demands a different kind of decisionmaking. Armed Forces Journal, 149(3), 26-28.

Corrigan, R. (2007). Digital Decision Making: Back to the Future: Springer Verlag.

Devlin, K. J. (2003). Logic and Information. Cambridge: Cambridge University Press.
Dibb, S., Meadows, M., \& Wilson, D. (2015). Digitisation and Decision Making in the Boardroom. New Economic Models in the Digital Economy. Retrieved from http://www.nemode.ac.uk/wp-

Doumeingts, G., Vallespir, B., \& Chen, D. (2003). GRAI Grid Decisional Modelling. In P. Bernus, K. Mertins, \& G. Schmidt (Eds.), Handbook on Architectures of Information Systems (pp. 313-339). Heidelberg: Springer Verlag.

Fadeyi, M. O. (2017). The role of building information modeling (BIM) in delivering the sustainable building value. International Journal of Sustainable Built Environment 6, 711-722.

Fadok, D. S., Boyd, J., \& Warden, J. (1995). Air Power's Quest for Strategic Paralysis: Air University Press.

Forte Asset Services. (2019). Building Management System Facts and Terms. Retrieved from https://www.forteassetservices.com.au/buildingmanagement-systems-bms/facts-terms/

Frayssinet, L., Merlier, L., Kuznik, F., Hubert, J., Milliez, M., \& Roux, J. (2018). Modeling the heating and cooling energy demand of urban buildings at city scale. Renewable and Sustainable Energy Reviews, 81, 23182327.

Gandomi, A., \& Haider, M. (2015). Beyond the hype: Big data concepts, methods, and analytics. International Journal of Information Management, 35, 137-144.

Goranson, T., \& Cardier, B. (2013). A two-sorted logic for structurally modeling systems. Progress in Biophysics and Molecular Biology, 113, 141-178.

Greenhalgh, T., Howick, J., \& Maskrey, N. (2014). Evidence based medicine: a movement in crisis? British Medical Journal, 348, 3725.

Hazen, B. T., Boone, C. A., Ezell, J. D., \& Jones-Farme, A. D. q. f. d., predictive analytics, and bigdata in supply chain management: An introduction to the problem and suggestions for research and applications. Int J. Production Econ 154:72-80. (2014). Data quality for datascience, predictive analytics, and bigdata in supply 
chain management: An introduction to the problem and suggestions for research and applications. Int $J$. Production Econ, 154, 72-80.

Horitaa, F., de Albuquerquea, J., Marchezinid, V., \& Mendiondoc, E. (2016). Bridging the gap between decision-making and big data sources: Application of model-based framework to disaster management in Brazil. Decision Support Systems, 97, 12-22.

Inmon, W. H., Zachman, J. A., \& Geiger, G. J. (1997). Data Stores, Data Warehousing and the Zachman Framework. New York, NY 10011: McGraw-Hill.

Kendall, A., \& Gal, Y. (2017). What Uncertainties Do We Need in Bayesian Deep Learning for Computer Vision? In $31^{\text {st }}$ Conference on Neural Information Processing Systems (NIPS 2017). Long Beach, CA.

Kimball, R. (1996). The Data Warehouse Toolkit. New York, N.Y.: Wiley \& Sons.

Kościelniaka, H., \& Putoa, A. (2015). BIG DATA in decision making processes of enterprises. Procedia Computer Science, 65, 1052-1058.

Lawson, H. (2006). A journey through the systems landscape. London: College Publications.

Li, M., Zhang, Z., Hu, Z., \& 17., W. P. (2017). Big Datadriven Technology Innovation: Concept and Key Problems. In WHICEB 2017 Proceedings: AIS Electronic Library.

Madden, S. (2012). From Databases to Big Data. IEEE Internet Computing, 16(3), 4-6.

Marr, B. (2015). Big Data: Using Smart Big Data, Analytics and Metrics to Make Better Decisions and Improve Performance Wiley \& Sons.

Matte, G., \& Rizzi, S. (2009). A Survey on Temporal Data Warehousing. Int Journal of data Warehousing \& Mining, 5(1), 1-17.

Noran, O., \& Bernus, P. (2018). Improving Digital Decision Making Through Situational Awareness. In B. Anderssonm, B. Johansson, S. Carlsson, C. Barry, M. Lang, H. Linger, \& C. Schneider (Eds.), ISD: Designing Digitalization. Lund, Sweden: Lund University.

Nowak, P., Ksiazek, M., Draps, M., \& Zawistowski, J. (2016). Decision Making using of Building Information Modeling. Procedia Engineering, 153, 519-526.

Osinga, F. (2006). Science, strategy and war: The strategic theory of John Boyd. London, UK: Routledge.

Pope, C. (2003). Resisting evidence: the study of evidencebased medicine as a contemporary social movement. Health, 7, 267-282.

Rokoeei, S. (2015). Building Information Modeling in Project Management: Necessities, Challenges and Outcomes. (Proceedings of 4th International Conference on Leadership, Technology, Innovation and Business Management), 87-95.

Santanilla, M., Zhang, D. W., Althouse, B. M., \& Ayers, J. W. (2014). What Can Digital Disease Detection Learn from Google Flu Trends? American Journal of Preventive Medicine, 47(3), 341-347.

Santos, E. T. (2009). Building Information Modelling and Interoperability. Proceedings of the $13^{\text {th }}$ Congress of the Iberoamerican Society of Digital Graphics, Brazil.
SAS. (2015). From Data to Action: A Harvard Business Review Insight Center Report. Retrieved from http://www.idgconnect.com/

Satyanarayana, R. G., Srinivasu, R., Poorna Rao, M., \& Rikkula, S. R. (2010). Data Warehousing, Data Mining, OLAP and OLTP Technologies Are Essential Elements To Support Decision-Making Process In Industries. Int. Journal on Comp. Sci. and Eng, 2(9), 2865-2873.

Schmidt, M. T., Elezi, F., Tommelein, I. D., \& Lindemann, U. (2014, 9-12 Dec. 2014). Towards recursive plan-docheck-act cycles for continuous improvement. Paper presented at the 2014 IEEE International Conference on Industrial Engineering and Engineering Management.

Seen, A., \& Sinha, A. P. (2005). Comparison of Data Warehousing Methods. Comm ACM, 48(3), 79-84.

Szafranski, R. (1995). A Theory of Information Warfare. Airpower Journal, 9(1), 1-11.

Teliceanu, M., Golovanov, N., Lazaroiu, G., \& Dumbrava, V. (2017). The importance of building clusters trading in smart cities. International Conference on ENERGY and ENVIRONMENT (CIEM).

Van Nederveen, G. A., \& Tolman, F. P. (1992). Modelling multiple views on buildings. Automation in Construction, 1(3), 215-224.

Wang, W., \& Benbasat, I. (2008). Attributions of Trust in Decision Support Technologies: A Study of Recommendation Agents for E-Commerce. Journal of Management Information Systems, 24(4), 249-273. doi:10.2753/MIS0742-1222240410

Wei, T., \& Zhu, Q. (2016, 22-25 May 2016). Co-scheduling of flexible energy loads in building clusters. Paper presented at the 2016 IEEE International Symposium on Circuits and Systems (ISCAS).

Zdravković, M., Noran, O., \& Trajanović, M. (2014). Towards Sensing Information Systems. In Proceedings of $23^{\text {rd }}$ International Conference on Information Systems (ISD2014). Berlin: Springer Verlag.

Zhang, X., Lovati, M., Vigna, I., Widén, J., Han, M., Gal, C., \& Feng, T. (2018). A review of urban energy systems at building cluster level incorporating renewable-energy-source (RES) envelope solutions. Applied Energy, 230, 1034-1056. 